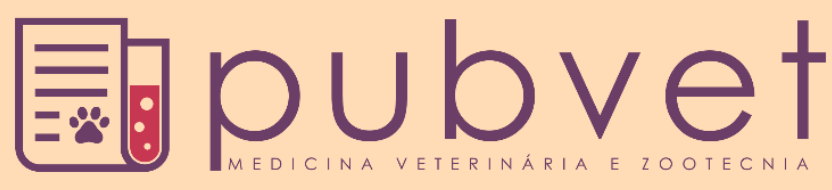

https://doi.org/10.31533/pubvet.v15n05a825.1-7

\title{
Espessamento miocárdico transitório em gato positivo para Bartonella spp.: Relato de Caso
}

\author{
Thaís Ribeiro Penna Paiva ${ }^{1^{*} \bullet}$, Anna Victória Antunes ${ }^{1 \bullet} \mathbb{0}$, Leonardo Bianchi de Oliveira ${ }^{1 \bullet}$, \\ Bruna Natali da Costa ${ }^{2} \odot$ (D), Simone Tostes de Oliveira Stedile ${ }^{3} \bullet$ (1), Thállitha Samih Wischral

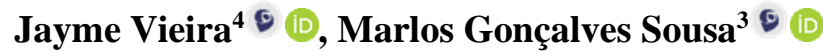 \\ ${ }^{I}$ Médica Veterinária Residente de Clínica Médica de Pequenos Animais, Hospital Veterinário da Universidade Federal do Paraná. Curitiba - PR, Brasil \\ ${ }^{2}$ Doutoranda em Ciência Veterinária da Universidade Federal do Paraná, Departamento de Medicina Veterinária. Curitiba - PR, Brasil \\ ${ }^{3}$ Professor (a) da Universidade Federal do Paraná, Departamento de Medicina Veterinária. Curitiba - PR, Brasil. \\ ${ }^{4}$ Médica Veterinária, Doutora, Laboratório de Doenças Transmitidaspor Vetores, Universidade Federal do Paraná, Departamentode Medicina Veterinária. Curitiba-PR, Brasil. \\ *Autor para correspondência, E-mail: thaisrppaiva@gmail.com
}

\begin{abstract}
Resumo. Um gato de dois anos de idade, sem raça definida, foi atendido por manifestar inapetência e apatia. Ao exame físico foram observados taquipneia, febre e sopro cardíaco. $\mathrm{O}$ animal apresentava também puliciose intensa e histórico de livre acesso à rua. $\mathrm{O}$ exame ecocardiográfico evidenciou espessamento miocárdico no ventrículo esquerdo. A partir de amostra de sangue, foi realizada reação em cadeia da polimerase (PCR) para detecção de Bartonella spp., com resultado positivo. Foi realizado ainda o teste rápido (ELISA) para o vírus da imunodeficiência felina (FIV) e vírus da leucemia felina (FELV), sendo ambos negativos. O gato foi tratado por 30 dias com doxiciclina (10 mg/kg, VO, BID), mostrando boa recuperação. Após 1 ano e 4 meses do término do tratamento, um novo ecocardiograma foi realizado e revelou o ventrículo esquerdo com espessura normal, o que sugere que o gato apresentou um espessamento miocárdico transitório (EMT), por provável cardiomiopatia inflamatória, decorrente da infecção pela Bartonella spp. A bartonelose, apesar de ser uma doença pouco investigada na rotina clínica, deve ser levada em consideração no diagnóstico diferencial em gatos jovens com espessamento do miocárdio, principalmente naqueles com sinais clínicos compatíveis.
\end{abstract}

Palavras chave: Cardiomiopatia inflamatória, bartonelose, miocardite

\section{Transient myocardial thickening in a cat positive for Bartonella spp.: Case report}

Abstract. A two-year-old, mixed-breed cat was attended for his lack of appetite and apathy. Physical examination revealed tachypnea, fever and heart murmur. The animal also had intense puliciosis and had historical of free access to the street. The echocardiographic examination showed myocardial thickening in the left ventricle. From a blood sample, a polymerase chain reaction (PCR) was performed to detect Bartonella spp. with a positive result. The rapid test (ELISA) was also performed for the feline immunodeficiency virus (FIV) and feline leukemia virus (FELV), both of which are negative. The cat was treated for 30 days with doxycycline $(10 \mathrm{mg} / \mathrm{kg}$, VO, BID), showing good recovery. After 1 year and 4 months of the end of treatment, a new echocardiogram was performed and revealed a normal thickness left ventricle, which suggests that the cat had a transient myocardial thickening (EMT), due to probable inflammatory cardiomyopathy, due to Bartonella spp. infection. Bartonellosis, despite being a disease little investigated in the clinical routine, needs to be taken into account in the differential diagnosis in young cats with myocardial thickening, especially in those with compatible clinical signs.

Keywords: Bartonellosis, inflammatory cardiomyopathy, miocarditis 


\section{Introdução}

A bartonelose é uma enfermidade causada por bacilos gram-negativos, aeróbicos, intraeritrocitários, que acometem diversas espécies e têm potencial zoonótico, causando, nas pessoas, a doença da arranhadura do gato. Dentre os gêneros mais comuns que infectam gatos estão a Bartonella henselae e, em menor proporção, a Bartonella clarridgeiae, onde o gato é a principal espécie portadora assintomática (Chomel et al., 2009; Clarridge et al., 1995). O mecanismo de contaminação no gato ocorre por meio de saliva e fezes de pulgas contaminadas da espécie Ctenocephalides felis durante o repasto sanguíneo (Chomel et al., 2009; Mikolajczyk \& O'Reilly, 2000).

Gatos com idade inferior a 12 meses, provenientes de abrigos, com livre acesso à rua, machos, não castrados e que possuam infestação de pulgas representam a maior parte da espécie contaminada (Cruz, 2018; Fleischman et al., 2015; Souza et al., 2010; Oliveira, 2018). Estudos conduzidos no Rio de Janeiro, São Paulo e Bahia, publicados em 2010, 2016 e 2018, respectivamente, demonstraram a alta prevalência sorológica e por detecção molecular, de Bartonella spp. e Bartonella henselae em gatos no Brasil (Santos et al., 2010; Souza et al., 2010; Cruz, 2018).

É observado que a maioria dos gatos infectados são assintomáticos e apresentam bacteremia persistente. Entretanto, nos sintomáticos, os principais sinais clínicos observados são letargia, febre, anorexia, linfadenomegalia, alterações oftálmicas, neurológicas, endocardite e, em menor escala, miocardite (Kordick et al., 1999; Mikolajczyk \& O’Reilly, 2000). O diagnóstico da Bartonella spp. pode ser feito por detecção sorológica, molecular ou microbiológica pela cultura sanguínea. Para o tratamento, pode ser utilizado azitromicina ou doxiciclina, nas doses de $10 \mathrm{mg} / \mathrm{kg}$ uma vez ao dia por 21 dias e de 10 a $22 \mathrm{mg} / \mathrm{kg}$ duas vezes ao dia por 14 a 28 dias, respectivamente.

Foram encontrados apenas dois estudos sobre gatos positivos para Bartonella spp. e que apresentaram espessamento miocárdico transitório (EMT) (Joseph et al., 2018; Novo Matos et al., 2018), não sendo relatados casos no Brasil. Desta forma, o objetivo do presente estudo é relatar o caso de um gato com EMT, positivo para Bartonella spp., incluindo seus sinais clínicos, assim como o diagnóstico e tratamento da doença.

\section{Relato de caso}

Foi atendido um gato, sem raça definida, pesando $4,4 \mathrm{~kg}$, castrado, de dois anos de idade, adotado há um ano e meio da rua, apresentando inapetência e apatia há dois dias. Na anamnese a tutora relatou que o animal não possuía comorbidades; porém, tinha livre acesso à rua, retornando, em diversas ocasiões, com lesões por arranhadura. $\mathrm{O}$ gato nunca havia sido vacinado e nem testado para o vírus da imunodeficiência felina (FIV) ou vírus da leucemia felina (FELV). Ao exame físico, apresentava taquipneia, ausculta cardíaca evidenciando sopro de grau $3 / 6 \mathrm{em}$ focos mitral e tricúspide, temperatura retal de $41,2^{\circ} \mathrm{C}$ e puliciose intensa.

O paciente foi admitido no internamento para estabilização, iniciando com o resfriamento corporal com toalhas úmidas e dose única de dipirona $25 \mathrm{mg} / \mathrm{kg}$ por via intravenosa (IV). A frequência respiratória diminuiu conforme a normalização da temperatura retal, variando de 64 movimentos por minuto $(\mathrm{mpm})$ na primeira hora, até $34 \mathrm{mpm}$ na terceira, momento no qual a temperatura se estabilizou em $39^{\circ}$ C. Os demais parâmetros, como pressão arterial sistólica, frequência cardíaca, mucosas, pulso e glicemia permaneceram sem alterações e estáveis.

Em seguida foram realizados exames laboratoriais de hemograma, bioquímica sérica, urinálise e teste de FIV e FELV. No hemograma, foi observada leucocitose $(19.700 / \mu 1$ [5.500 - 19.500/ $\mu 1])$ por neutrofilia $(16.745 / \mu 1 \quad[2.500-12.500 / \mu 1])$. As avaliações bioquímicas realizadas (alanina aminotransferase, fosfatase alcalina, gamaglutamiltransferase, ureia, creatinina, lactato, e proteínas totais e frações) estavam todas dentro do limite de referência, bem como a urinálise. Além disso, o paciente testou negativo para FIV e FELV (Snap FIV/FELV Combo Test, Idexx ${ }^{\circledR}$ ).

Tendo em vista a clínica do paciente de febre e leucocitose, foi iniciada terapia antibiótica empírica de amplo espectro com ampicilina com sulbactam $20 \mathrm{mg} / \mathrm{kg}$ TID por via IV e o paciente foi mantido na fluidoterapia com Ringer Lactato na taxa de manutenção de $50 \mathrm{ml} / \mathrm{kg} / \mathrm{dia}$, apenas para viabilizar o acesso venoso. A fim de complementar a investigação do sopro apresentado pelo paciente, foi realizado um 
ecocardiograma, no qual pode-se observar que a espessura do ventrículo esquerdo (VE) e do septo interventricular, mensuradas em modo $\mathrm{M}$, bem como a espessura do VE na via de saída ao final da diástole apresentaram-se acima dos valores de referência para a espécie (Tabela 1). Além disso, a relação átrio esquerdo/aorta (AE/Ao) estava em limite superior, o átrio esquerdo (AE) estava próximo ao limite superior e o paciente possuía discreta quantidade de efusão pericárdica, sem tamponamento cardíaco. Também se observou movimento anterior sistólico da válvula mitral e regurgitação discreta em válvulas mitral, tricúspide e pulmonar. O exame eletrocardiográfico evidenciou ritmo sinusal, sem alterações.

Tabela 1. Mensurações em Modo M do ecocardiograma pré e pós-tratamento com doxiciclina em um gato com bartonelose. Constam na tabela os valores em milímetros $(\mathrm{mm})$ do: septo e parede do ventrículo esquerdo em sístole e em diástole, espessura do ventrículo esquerdo na via de saída ao final da diástole e a relação átrio esquerdo/aorta.

\begin{tabular}{|c|c|c|c|c|c|}
\hline \multicolumn{2}{|l|}{ Parâmetros } & $\begin{array}{c}\text { Medidas } \\
\text { Pré tratamento }\end{array}$ & $\begin{array}{c}\text { Medidas } \\
\text { Pós-tratamento }\end{array}$ & $\begin{array}{c}\text { Valor de referência } \\
\text { (Gatos } \leq 4,5 \mathrm{~kg} \text { ) }\end{array}$ & $\begin{array}{c}\text { Referências } \\
\text { Bibliográficas }\end{array}$ \\
\hline \multirow{2}{*}{ Ventrículo esquerdo em sístole } & Septo & $9,3 \mathrm{~mm}$ & $7 \mathrm{~mm}$ & $\leq 8,7 \mathrm{~mm}$ & \multirow{4}{*}{$\begin{array}{l}\text { Häggström et al. } \\
\qquad \underline{(2016)}\end{array}$} \\
\hline & Parede & $9,3 \mathrm{~mm}$ & $7,4 \mathrm{~mm}$ & $\leq 8,7 \mathrm{~mm}$ & \\
\hline \multirow{2}{*}{ Ventrículo esquerdo em diástole } & Septo & $5,2 \mathrm{~mm}$ & $4 \mathrm{~mm}$ & $\leq 5,1 \mathrm{~mm}$ & \\
\hline & Parede & $6,1 \mathrm{~mm}$ & $4,3 \mathrm{~mm}$ & $\leq 5 \mathrm{~mm}$ & \\
\hline \multirow{3}{*}{\multicolumn{2}{|c|}{$\begin{array}{l}\text { Espessura do ventrículo esquerdo na via de } \\
\text { saída ao final da diástole } \\
\text { Átrio esquerdo } \\
\text { Relação átrio esquerdo/aorta }\end{array}$}} & $6,2 \mathrm{~mm}$ & NR & $\leq 6 \mathrm{~mm}$ & Wagner et al. $(\underline{2010})$ \\
\hline & & $14 \mathrm{~mm}$ & $12 \mathrm{~mm}$ & $8,2-14,5 \mathrm{~mm}$ & Häggström et al. \\
\hline & & 1,4 & 1,33 & $0,88-1,43$ & $(\underline{2016})$ \\
\hline
\end{tabular}

NR: não realizado.

Devido a idade do paciente, sinais clínicos, leucocitose por neutrofilia, contato com animais errantes, puliciose e o espessamento do ventrículo esquerdo, incluiu-se a bartonelose como possível diagnóstico. Uma alíquota de sangue com EDTA, coletada no primeiro dia de internamento e antes da antibioticoterapia, foi encaminhada ao laboratório de biologia molecular para pesquisa de DNA da Bartonella spp.

A extração de DNA foi realizada utilizando o kit comercial ReliaPrep ${ }^{\mathrm{TM}}$ Blood gDNA Miniprep System (Promega Corporation, Madison, USA), de acordo com as recomendações do fabricante. Para verificar a presença de inibidores nas amostras de DNA, foi realizado uma PCR convencional para o gene Glyceraldehyde 3-phosphate dehydrogenase (GAPDH), de acordo com protocolos previamente descritos (Birkenheuer et al., 2003). Para a detecção de DNA de Bartonella spp., foi realizada uma cPCR, utilizando os pares de primers 325s (5'-CTT CAG ATG ATG ATC CCAAGC CTT YTG GCG -3') e 1100as (5'- GAA CCG ACG ACC CCC TGC TTGCAA AGC A-3'), que amplificam 453-717 pares de base da região intergênica do16S-23S do RNA ribossomal (ITS), conforme protocolo previamente descrito (Maggi \& Breitschwerdt, 2005).

No segundo dia de internação o gato voltou a apresentar febre de $39,4^{\circ} \mathrm{C}$, momento em que foi administrada mais uma dose de dipirona de $12,5 \mathrm{mg} / \mathrm{kg}$ por via IV, contudo, nos 3 dias subsequentes, todos os parâmetros se mantiveram estáveis, o paciente voltou a se alimentar e a alta hospitalar foi concedida.

O resultado da reação em cadeia de polimerase (PCR), com a detecção do DNA da Bartonella spp., foi liberado no nono dia pós atendimento (Figura 1), confirmando a suspeita clínica. Em posse do resultado, o tratamento foi modificado, iniciando a terapia antibiótica com doxiciclina na dose de 10 $\mathrm{mg} / \mathrm{kg}$ BID, por via oral (VO), por 30 dias e selamectina pour on a cada 30 dias, para tratamento e prevenção da puliciose.

Após 4 dias recebendo doxiciclina, o paciente apresentou êmese e foi adicionado omeprazol $1 \mathrm{mg} / \mathrm{kg}$ BID por VO. Os retornos para acompanhamento foram realizados no $14^{\circ}$ dia de terapia com doxiciclina e dois dias após o fim da antibioticoterapia. Em ambos, não foi mais auscultado sopro, o paciente estava ativo, sem febre, se alimentando sozinho e não apresentava êmese.

Por fim, foi executado ecocardiograma de acompanhamento um ano e quatro meses após o fim do tratamento. As medidas do septo parede do VE, bem como a relação AE/Ao estavam dentro da referência para a espécie (Tabela 1). A valva tricúspide apresentou estrutura normal com função insuficiente e a valva pulmonar apresentou escape. Foi evidenciado enchimento ventricular com 
padrão de relaxamento anormal (tipo I). O exame eletrocardiográfico permaneceu sem alterações, com ritmo sinusal.

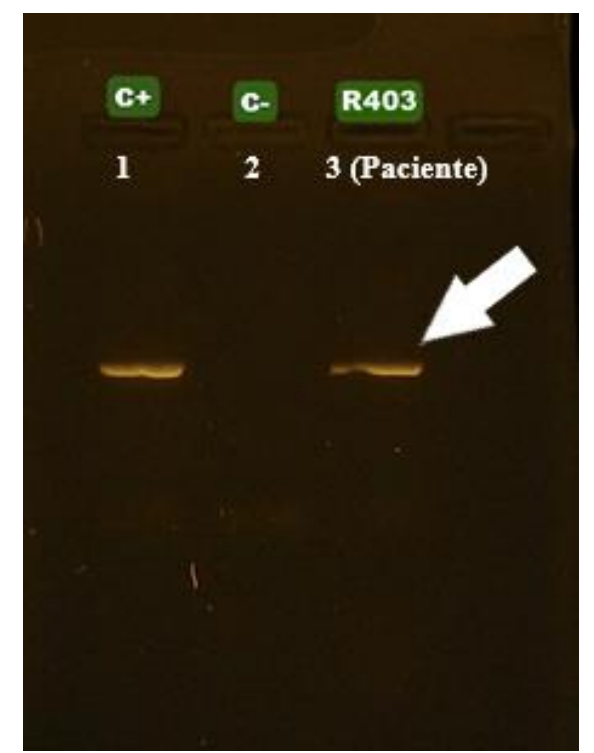

Figura 1. Eletroforese em gel de agarose 1,5\%, corado com sybr safe (Invitrogen, CA, USA). Canaleta 1: controle positivo (C+: Bartonella spp.), canaleta 2: controle negativo (C-: água ultra-pura esterilizada) e canaleta 3: resultado positivo para Bartonella spp. do paciente (R403) (seta branca).

\section{Discussão}

O presente relato apresenta um gato positivo para Bartonella spp., com espessamento miocárdico transitório (EMT), tendo resolução dos sinais clínicos e ecocardiográficos após o tratamento de quatro semanas com doxiciclina.

O paciente discutido era jovem e com livre acesso à rua e infestado por pulgas, o que corrobora com o descrito na literatura, na qual a maior proporção de gatos contaminados eram machos, tinham, em média, um ano de idade, eram provenientes de abrigos, tinham livre acesso à rua e que possuíam infestação de pulgas (Cruz, 2018; Fleischman et al., 2015; Souza et al., 2010). Apesar do gato relatado ser castrado, em várias ocasiões apresentava lesões de briga após retornar da rua, visto que podia ter contato sem supervisão com outros gatos, o que justifica sua propensão a contaminação, considerando o meio de transmissão da doença.

Os sinais clínicos apresentados pelo paciente foram inapetência, taquipneia, febre, sopro, miocardite e, corroborando com os demais relatos (Breitschwerdt, 2008; Joseph et al., 2018; Kordick et al., 1999; Mikolajczyk \& O'Reilly, 2000; Novo Matos et al., 2018). Embora geralmente gatos com bartonelose sejam assintomáticos, os sinais clínicos apresentados pelo paciente foram cruciais para a suspeita e diagnóstico da doença (Kordick et al., 1999). Apesar de não haver relatos de alterações hematológicas específicas relacionadas à infecção por bactérias do gênero Bartonella em gatos, o paciente apresentava uma leve leucocitose por neutrofilia. Foi descrito por Cruz (2018) que a grande parte dos gatos positivos para Bartonella spp. apresentam uma leve linfopenia e hiperproteinemia, entretanto, nesse relato foi observada apenas uma discreta leucocitose por neutrofilia antes do tratamento ser iniciado. Devido a suspeita de miocardite, o leucograma apresentado pelo paciente poderia ser em decorrência dessa alteração.

A ocorrência de miocardite, ou cardiomiopatia inflamatória, em cães e gatos, devido a infecção por Bartonella henselae já foi descrito por Kordick et al. (1999), Nakamura et al. (2011) e Varanat et al. (2012). Relatos de EMT em gatos decorrentes da infecção por Bartonella henselae foram descritos apenas por Joseph et al. (2018) e Novo Matos et al. (2018). A patofisiologia do EMT ainda não foi bem elucidada, entretanto, no estudo feito por Novo Matos et al. (2018), em 21 gatos com EMT, especulou- 
se que a provável causa seria um edema agudo em decorrência da infiltração celular no miocárdio, o que torna a miocardite a principal suspeita de resultar no EMT. No entanto, neste mesmo estudo, apenas quatro gatos foram investigados para infecção por Bartonella Henselae, sendo que desses, três apresentaram sorologia positiva, sugerindo que a doença pode estar sendo subestimada como causa de EMT em gatos.

Outras causas infecciosas de espessamento miocárdico em gatos, também descritas, são a FIV, o vírus da panleucopenia felina e infecção por Toxoplasma gondii (Meurs et al., 2000; Novo Matos et al., 2018; Rolim et al., 2016; Simpson et al., 2005). Apesar de não terem sido feitos testes para toxoplasmose e panleucopenia felina, o paciente não apresentava evidências em exames hematológicos que sugerissem tais infecções. No estudo de Novo Matos et al. (2018), os quatro gatos com EMT testados para B. henselae foram também testados para $T$. gondii, sendo todos negativos para o segundo patógeno.

Um impasse reportado em pesquisas semelhantes é a confrontação entre a cardiomiopatia hipertrófica $(\mathrm{CMH})$ e o EMT. Um dos principais quesitos é a idade do gato, visto que a CMH é mais frequente em animais de idade avançada, ao passo que o EMT é mais relatado entre 1,7 a 2 anos de vida (Novo Matos et al., 2018; Payne et al., 2015; Rush et al., 2002). Além disso, conforme observado por Novo Matos et al. (2018), os casos de EMT possuíam a parede do ventrículo esquerdo mais fina do que aqueles com CMH. A média de espessamento de parede do VE, nos casos de EMT, era de $6,8 \mathrm{~mm}$, próximo ao observado neste estudo $(6,2 \mathrm{~mm})$, já nos casos de $\mathrm{CMH}$ a média era de $8,1 \mathrm{~mm}$. Outras duas avaliações relevantes são a razão $\mathrm{AE} / \mathrm{AO}$ e o tamanho do átrio esquerdo (AE), que nesse relato estavam, respectivamente, em limite superior e próximo a ele, tendo seus valores normalizados após o tratamento com doxiciclina. Tal fato foi observado também por Novo Matos et al. (2018) e Joseph et al. (2018), onde ambas as medidas reduziram após o tratamento. Comparativamente, nos casos de CMH as duas mensurações tenderam a aumentar com o passar do tempo (Novo Matos et al., 2018). Sendo assim, é possível perceber que o acompanhamento ecocardiográfico do paciente é o melhor método para distinguir ambas as enfermidades, visto que no EMT há resolução dos sinais ecocardiográficos, ao passo que na CMH o quadro é progressivo. A infecção por Bartonella spp. deveria ser avaliada nos casos de espessamento de parede e septo ventricular, principalmente em gatos jovens, pois parece ser um diagnóstico diferencial importante nestes casos.

O diagnóstico definitivo de miocardite, tanto na medicina quanto na medicina veterinária, é feito através de exame histopatológico, seja antemortem por biópsia endomiocárdica ou posmortem por necropsia (Caforio et al., 2013). Uma vez que o paciente sobreviveu e devido à dificuldade de se executar tal técnica em gatos, não foi possível realizar a biópsia e o tratamento seguiu com base na provável causa. Para o diagnóstico clínico da miocardite foi levado em consideração os critérios necessários para a identificação de miocardite em cães (Lakhdhir et al., 2020), uma vez que não existem tais critérios estabelecidos para gatos. No presente estudo, o paciente preencheu um critério maior de diagnóstico de miocardite, por ter a detecção molecular de um agente causador e quatro critérios menores, sendo a febre, o surgimento de sopro cardíaco, leucograma inflamatório e efusão pericárdica. Desta forma, seguindo a analogia do estudado em cães, no qual é necessário que seja cumprido um critério maior e no mínimo 3 menores, o diagnóstico seria definitivo para o caso relatado.

Por fim, o padrão de relaxamento anormal do tipo 1 durante o enchimento ventricular observado no segundo ecocardiograma, o qual caracteriza uma disfunção diastólica, poderia ser decorrente de uma fibrose ocasionada pela inflamação do miocárdio, bem como poderia ser um sinal de CMH (Fuentes, 2003). Nesse relato, conforme discutido, devido à ausência de sinais que corroborem com o diagnóstico de $\mathrm{CMH}$, a provável causa seria uma sequela da injúria sofrida pelo miocárdio.

\section{Conclusão}

O presente trabalho descreve o primeiro relato de caso do Brasil de EMT associada a infecção por Bartonella spp. O diagnóstico da EMT depende de ecocardiogramas seriados, porém, ao primeiro exame no qual o espessamento da parede e/ou do septo ventricular seja observado, a bartonelose deve ser levada em consideração como diagnóstico diferencial, principalmente em pacientes jovens. 


\section{Referências}

Birkenheuer, A. J., Levy, M. G., \& Breitschwerdt, E. B. (2003). Development and evaluation of a seminested PCR for detection and differentiation of Babesia gibsoni (Asian genotype) and B. canis DNA in canine blood samples. Journal of Clinical Microbiology, 41(9), 4172-4177. https://doi.org/10.1128/JCM.41.9.4172-4177.2003.

Breitschwerdt, E. B. (2008). Feline bartonellosis and cat scratch disease. Veterinary Immunology and Lmmunopathology, 123(1-2), 167-171. https://doi.org/10.1016/j.vetimm.2008.01.025.

Caforio, A. L. P., Pankuweit, S., Arbustini, E., Basso, C., Gimeno-Blanes, J., Felix, S. B., Fu, M., Heliö, T., Heymans, S., \& Jahns, R. (2013). Current state of knowledge on aetiology, diagnosis, management, and therapy of myocarditis: a position statement of the European Society of Cardiology Working Group on Myocardial and Pericardial Diseases. European Heart Journal, 34(33), 26362648. https://doi.org/10.1093/eurheartj/eht210.

Chomel, B. B., Boulouis, H.-J., Breitschwerdt, E. B., Kasten, R. W., Vayssier-Taussat, M., Birtles, R. J., Koehler, J. E., \& Dehio, C. (2009). Ecological fitness and strategies of adaptation of Bartonella species to their hosts and vectors. Veterinary Research, 40(2), 1-22. https://doi.org/10.1051/vetres/2009011.

Clarridge, J. E., Raich, T. J., Pirwani, D., Simon, B., Tsai, L., Rodriguez-Barradas, M. C., Regnery, R., Zollo, A., Jones, D. C., \& Rambo, C. (1995). Strategy to detect and identify Bartonella species in routine clinical laboratory yields Bartonella henselae from human immunodeficiency virus-positive patient and unique Bartonella strain from his cat. Journal of Clinical Microbiology, 33(8), 21072113. https://doi.org/10.1128/JCM.33.8.2107-2113.

Cruz, T. A. O. (2018). Ocorrência de Bartonella spp. no sangue e unhas de felinos domésticos da região sul da Bahia - avaliação dos fatores de risco, perfil epidemiológico e caracterização molecular. Universidade Estadual de Santa Cruz.

Fleischman, D. A., Chomel, B. B., Kasten, R. W., Stuckey, M. J., Scarlet, J., Liu, H., Boulouis, H.-J., Haddad, N., \& Pedersen, N. C. (2015). Bartonella infection among cats adopted from a San Francisco shelter, revisited. Applied and Environmental Microbiology, 81(18), 6446-6450. https://doi.org/10.1128/AEM.01864-15.

Fuentes, V. L. (2003). Diastolic function - is this the key to successful management of many feline cardiomyopathies? Journal of Feline Medicine and Surgery, 5(1), 51-56. https://doi.org/10.1053/jfms.2002.0200.

Häggström, J., Andersson, Å. O., Falk, T., Nilsfors, L., OIsson, U., Kresken, J. G., Höglund, K., Rishniw, M., Tidholm, A., \& Ljungvall, I. (2016). Effect of body weight on echocardiographic measurements in 19,866 pure-bred cats with or without heart disease. Journal of Veterinary Internal Medicine, 30(5), 1601-1611. https://doi.org/10.1111/jvim.14569.

Joseph, J. L., Oxford, E. M., \& Santilli, R. A. (2018). Transient myocardial thickening in a Bartonella henselae-positive cat. Journal of Veterinary Cardiology, 20(3), 198-203. https://doi.org/10.1016/j.jvc.2018.04.003.

Kordick, D. L., Brown, T. T., Shin, K., \& Breitschwerdt, E. B. (1999). Clinical and pathologic evaluation of chronic bartonella henselae or bartonella clarridgeiae infection in cats. Journal of Clinical Microbiology, 37(5), 1536-1547. https://doi.org/10.1128/JCM.37.5.1536-1547.1999.

Lakhdhir, S., Viall, A., Alloway, E., Keene, B., Baumgartner, K., \& Ward, J. (2020). Clinical presentation, cardiovascular findings, etiology, and outcome of myocarditis in dogs: 64 cases with presumptive antemortem diagnosis (26 confirmed postmortem) and 137 cases with postmortem diagnosis only (2004-2017). Journal of Veterinary Cardiology, 30, 44-56. https://doi.org/10.1016/j.jvc.2020.05.003.

Maggi, R. G., \& Breitschwerdt, E. B. (2005). Potential limitations of the 16S-23S rRNA intergenic region for molecular detection of Bartonella species. Journal of Clinical Microbiology, 43(3), 11711176. https://doi.org/10.1128/JCM.43.3.1171-1176.2005.

Meurs, K. M., Fox, P. R., Magnon, A. L., Liu, S.-K., \& Towbin, J. A. (2000). Molecular screening by polymerase chain reaction detects panleukopenia virus DNA in formalin-fixed hearts from cats with 
idiopathic cardiomyopathy and myocarditis. Cardiovascular Pathology, 9(2), 119-126. https://doi.org/10.1016/s1054-8807(00)00031-4.

Mikolajczyk, M. G., \& O'Reilly, K. L. (2000). Clinical disease in kittens inoculated with a pathogenic strain of Bartonella henselae. American Journal of Veterinary Research, 61(4), 375-379. https://doi.org/10.2460/ajvr.2000.61.375.

Nakamura, R. K., Zimmerman, S. A., \& Lesser, M. B. (2011). Suspected Bartonella-associated myocarditis and supraventricular tachycardia in a cat. Journal of Veterinary Cardiology, 13(4), 277281. https://doi.org/10.1016/j.jvc.2011.08.004.

Novo Matos, J., Pereira, N., Glaus, T., Wilkie, L., Borgeat, K., Loureiro, J., Silva, J., Law, V., Kranjc, A., \& Connolly, D. J. (2018). Transient myocardial thickening in cats associated with heart failure. Journal of Neterinary Internal Medicine, 32(1), 48-56. https://doi.org/10.1111/jvim.14897.

Oliveira, D. E. A. (2018). Ocorrência de Bartonella spp. no sangue e unhas de felinos domésticos da região sul da Bahia - avaliação dos fatores de risco, perfil epidemiológico e caracterização molecular. Tese (Doutorado em Ciência Animal) - Universidade Estadual de Santa Cruz. Bahia, 61 p.

Payne, J. R., Brodbelt, D. C., \& Fuentes, V. L. (2015). Cardiomyopathy prevalence in 780 apparently healthy cats in rehoming centres (the CatScan study). Journal of Veterinary Cardiology, 17, S244S257. https://doi.org/10.1016/j.jvc.2015.03.008.

Rolim, V. M., Casagrande, R. A., Wouters, A. T. B., Driemeier, D., \& Pavarini, S. P. (2016). Myocarditis caused by feline immunodeficiency virus in five cats with hypertrophic cardiomyopathy. Journal of Comparative Pathology, 154(1), 3-8. https://doi.org/10.1016/j.jcpa.2015.10.180.

Rush, J. E., Freeman, L. M., Fenollosa, N. K., \& Brown, D. J. (2002). Population and survival characteristics of cats with hypertrophic cardiomyopathy: 260 cases (1990-1999). Journal of the American Veterinary Medical Association, 220(2), 202-207. https://doi.org/10.2460/javma.2002.220.202.

Santos, S. R. C., Drummond, M. R., Gilioli, R., Lania, B. G., \& Velho, P. E. N. F. (2010). Bacteremia por Bartonella Henselae em gatos não domiciliados de Campinas, sp. Sínteses: Revista Eletrônica Do SimTec, 3, 243-244. https://doi.org/10.20396/sinteses.v1i3.7824.

Simpson, K. E., Devine, B. C., \& Gunn-Moore, D. (2005). Suspected toxoplasma-Associated myocarditis in a cat. Journal of Feline Medicine and Surgery, 7(3), 203-208. https://doi.org/10.1016/j.jfms.2004.08.004.

Souza, A. M., Almeida, D. N. P., Guterres, A., Gomes, R., Favacho, A. R. M., dos Santos Moreira, N., Maia, L. M. P., Rozental, T., Torres Filho, R. A., \& Cerqueira, A. de M. F. (2010). Bartonelose: análise molecular e sorológica em gatos do Rio de Janeiro-Brasil. Revista Brasileira de Ciência Veterinária, 17(1), 7-11. https://doi.org/10.4322/rbcv.2014.135.

Varanat, M., Broadhurst, J., Linder, K. E., Maggi, R. G., \& Breitschwerdt, E. B. (2012). Identification of Bartonella henselae in 2 cats with pyogranulomatous myocarditis and diaphragmatic myositis. Veterinary Pathology, 49(4), 608-611. https://doi.org/10.1177/0300985811404709.

Wagner, T., Fuentes, V. L., Payne, J. R., McDermott, N., \& Brodbelt, D. (2010). Comparison of auscultatory and echocardiographic findings in healthy adult cats. Journal of Veterinary Cardiology, 12(3), 171-182. https://doi.org/10.1016/j.jvc.2010.05.003.

\section{Histórico do artigo:}

Recebido: 11 de janeiro de 2021

Aprovado: 4 de fevereiro de 2021 .

Disponível online: 30 de abril de 2021.
Licenciamento: Este artigoé publicado na modalidade Acesso Aberto sob a licença Creative Commons Atribuição 4.0 (CC-BY 4.0), a qual permite uso irrestrito, distribuição, reprodução em qualquer meio, desde que $o$ autore a fonte sejam devidamente creditados. 